\title{
SOFT SYSTEMS METHODOLOGY AND SIMULATION MODELING
}

\author{
Brian Lehaney \\ Faculty of Business \\ University of Luton \\ Luton, Beds, LU1 3J, UK
}

\author{
Ray J. Paul \\ Department of Computing and IS \\ Brunel University \\ Uxbridge, UB8 3PH, UK
}

\begin{abstract}
Simulation is both popular and powerful, but reportage of simulation case studies indicates that in many cases process is treated cursorily, and end-user-acceptance of final models is not forthcoming. Whilst texts often proclaim the importance of process, this is usually left to the discretion of the modeler. A range of problem structuring (or soft) methodologies have been developed to address process issues. However, these can be both slow and unwieldy. This paper outlines a case which utilises the principles of soft methodologies in a relatively quick and dirty approach to process.
\end{abstract}

\section{INTRODUCTION}

Simulation provides managers with a powerful means to assess the demands on resources created by variable patterns of arrivals and service rates, such as those experienced in hospital outpatients departments. Analytical techniques such as queuing theory may not be of help in such situations, if their assumptions are too rigid or unrealistic or the situation is too complex. However, simulation does not provide a panacea, and much depends upon the way in which it is used. In order to ascertain the appropriate system specification, and model parameters, and in order to assess the value of the simulation, process of discussion an debate must be undertaken. Indeed, that very discussion and debate may provide resolution in itself.

Despite the possibility of good intentions, simulation textbooks tend to ignore, or mention only fleetingly, the important processes of problem formulation and logical model development. As Paul and Balmer $(1993, \mathrm{p} 5)$ note 'Experience of this process of model formulation is not easy to provide in the context of the essentially artificial 'practical' exercises in either a textbook or academic course'. In fact simulation texts give no real guidance as to how this process should be undertaken.

In recent years problem structuring (soft methods) has

flourished as an area of academic and practitioner interest. A range of methodologies has been developed, including
Soft Systems Methodology (Checkland, 1981, Wilson, 1984). These draw from the following principles:

- emphasis on problem identification, problem structuring, and problem resolution, rather than on problem solution;

- acceptance of multiple problem-perspectives;

- belief that the researcher will affect the situation;

- consensus and participation rather than imposition;

- continual re-evaluation;

- no automatic acceptance of existing structures;

- involvement of those being researched in the research process;

- concern to elicit repressed or minority views;

- challenging approaches to 'norms'. Whilst the approaches vary in many ways, the arguments in their favour are that they provide:

- structured approaches to problem identification;

- increased sense of model ownership, and hence increased likelihood of model confidence;

- increased probability of implementing recommendations;

- a reference framework which may be particularly useful in tricky situations, especially for the less experienced analyst;

- improved communication through the use of a known modelling methodology;

- an important reminder of the process to authors who are writing up case material - process is often omitted in case articles.

Soft Systems Methodology is advocated as the most suitable for combining with simulation because:

- each approach provides important features missing from the other;

- the two can be linked in a truly complementary manner;

- SSM is probably the best known soft method;

- SSM can be used to undertake the important problem structuring phase of simulation, assisting in identifying system boundaries and system activities;

- SSM creates an activity-based model which can be converted to an activity cycle diagram;

- simulation depicts a range of options which assists in assessing their feasibility and desirability; 
- simulation produces dynamic models which can be used to investigate interactions;

- SSM enables 'hidden' activities to be identified, by means of issue-based root definitions;

- the use of SSM reduces the chance of implementation failure which results from misunderstandings.

\section{WHAT IS SOFT SYSTEMS METHODOLOGY?}

Soft systems methodology (SSM) is an approach to modelling developed by Checkland (1981) and Wilson (1984), as an alternative to traditional 'hard' approaches, which are based on the prescriptive use of techniques, for clearly defined problems. Such techniques are of little use in the initial analysis of a human activity system, which is likely to have problem areas that are not clearly identified, and which are unstructured. Soft system methodology enables the people involved in running a system (Actors), those responsible for controlling it (Owners), and those who receive its benefits (Customers), to participate in the process of developing a system model, which is likely to encourage acceptability of the model. SSM may be used to aid the identification of system boundaries and system activities, particularly in complex systems. This is particularly useful in a case where a 'hard' technique may eventually be applied, such as in the simulation of an out- patients system. Activity areas within the National Health Service (NHS) are typically 'messy', and particularly suited to an SSM approach, and to the application of simulation.

The general seven stages of soft systems methodology are shown in Figure 1. The methodology is iterative in approach, and is not prescriptive as to a starting point. The unstructured problem situation (1) is described by a rich picture (2). This is used to search for relevant systems which are described by a root definition (3) and conceptual model (4). Although other forms of root definition are possible, those which are both consensusbased, and issue-based are the most interesting and most useful. A root definition describes a system in terms of the following components.

Owners, the roles of monitoring and taking appropriate control actions;

Actors, the roles of running the system;

Customers, the beneficiaries (or victims) of the system;

Transformation, the system inputs converted to outputs;

Weltanschauung, the world view, the perspective taken in defining the system;

Environment, the constraints on the system.

The above can be formed as the mnemonic CATWOE, and may be used to deter the modeler from omitting important system elements. Any omissions should be deliberate and rational.

\section{A CONCEPTUAL COMBINATION OF SIMULATION WITH SSM}

Figure 2 shows how SSM and simulation may be combined. Phase 1 comprises the early stages of SSM, in which finding out about the problem situation is undertaken, the problem situation is expressed (rich picture), and the system is described (root definition). From the root definition, a conceptual model is formed which contains the minimum set of activities needed to support the root definition. The conceptual model is compared with the system (Phase 1a), and, where appropriate, the root definition is changed, and a new conceptual model developed. If the activity-level of the conceptual model is too broad, selected activities are expanded until the appropriate level of resolution is reached. As primary task root definitions are used, it is likely that the systems identified within the conceptual model will match those of the organisation. Following temporary entities through the system may result in systems which do not match those of the organisation and which may not have owners. At this stage, if such systems are identified, it may be that control actions are taken. If the organisation appoints an owner of a newlyidentified system, for example, this may be sufficient to address any problem situations which have arisen.

If Phase 7 indicates that model output and system output do not match as well as is desired, the route of change must be through Phase 4 , as adjusting the model ad hoc is likely to lead to self-fulfilling validation prophecies. (The modeler must make judgements regarding minor changes.) The route through Phase 4 preserves the integrity of the model, and mimics the 'Rich-Picture-to-Root-Defintion-to-Conceptual-Model-toRich-Picture-to-Root Definition' circle of SSM, which should not be broken by direct input from the Rich Picture to the Conceptual Model. (The latter should be built solely from the root definition.)

If the conceptual model development has been undertaken rigorously and with 'good' participation, it should be unnecessary to revisit the early SSM stages in the first iteration. These should, however, be revisited as part of the overall process, as once policy changes have been explored and implemented, the system, being dynamic, has led to another investigation. The cycle then continues.

The concept proposed is appealing in many ways, but it is not without its difficulties. As Mingers (1992) notes, despite Checkland's assurances that SSM is timeindependent, it is in fact time consuming. A useful approach is one which enables an acceptable simulation 
model to be built quickly, but within a 'quick and dirty' framework which embodies the principles of soft methodologies.

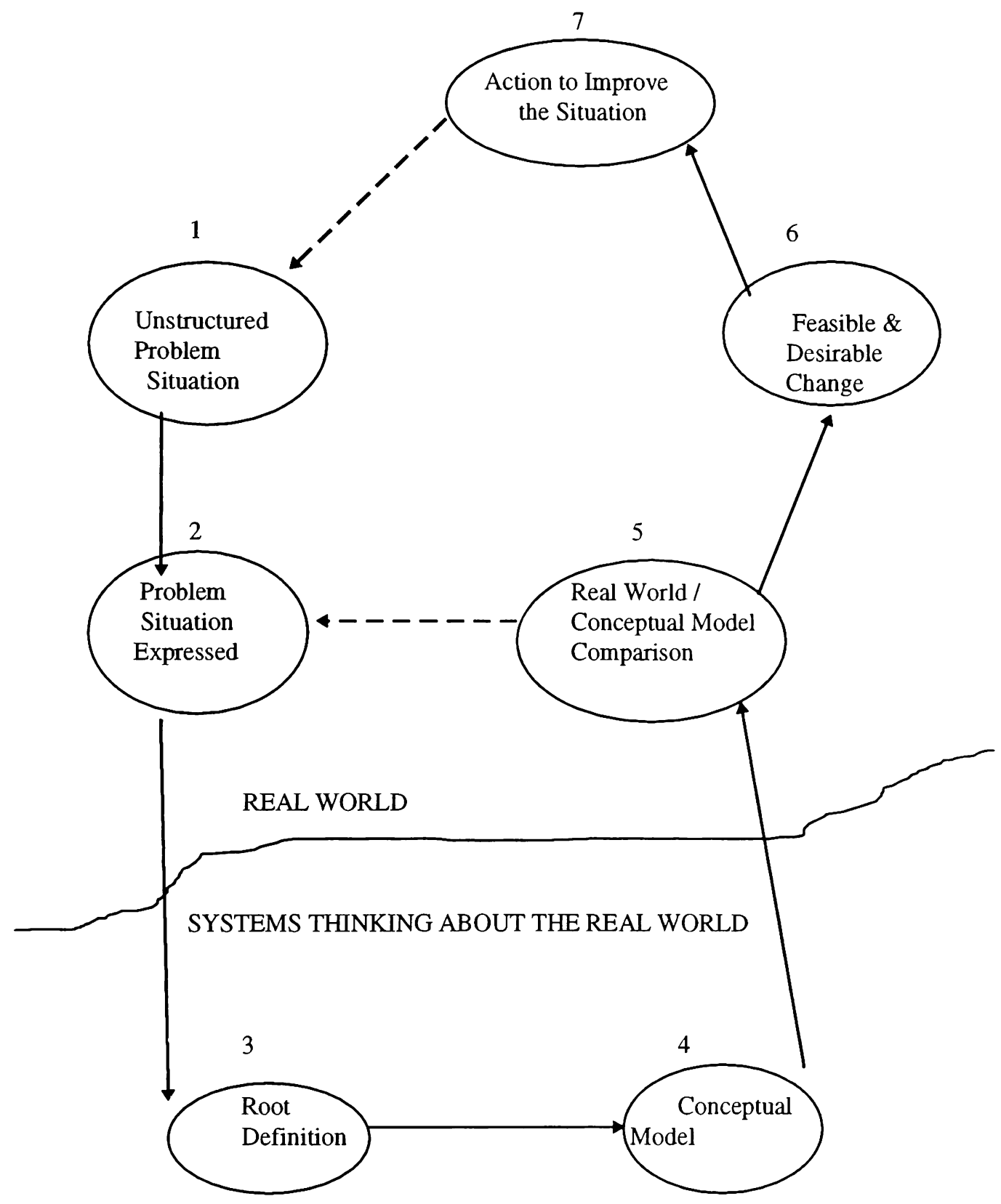

Figure 1: The SSM Seven Stage Process 


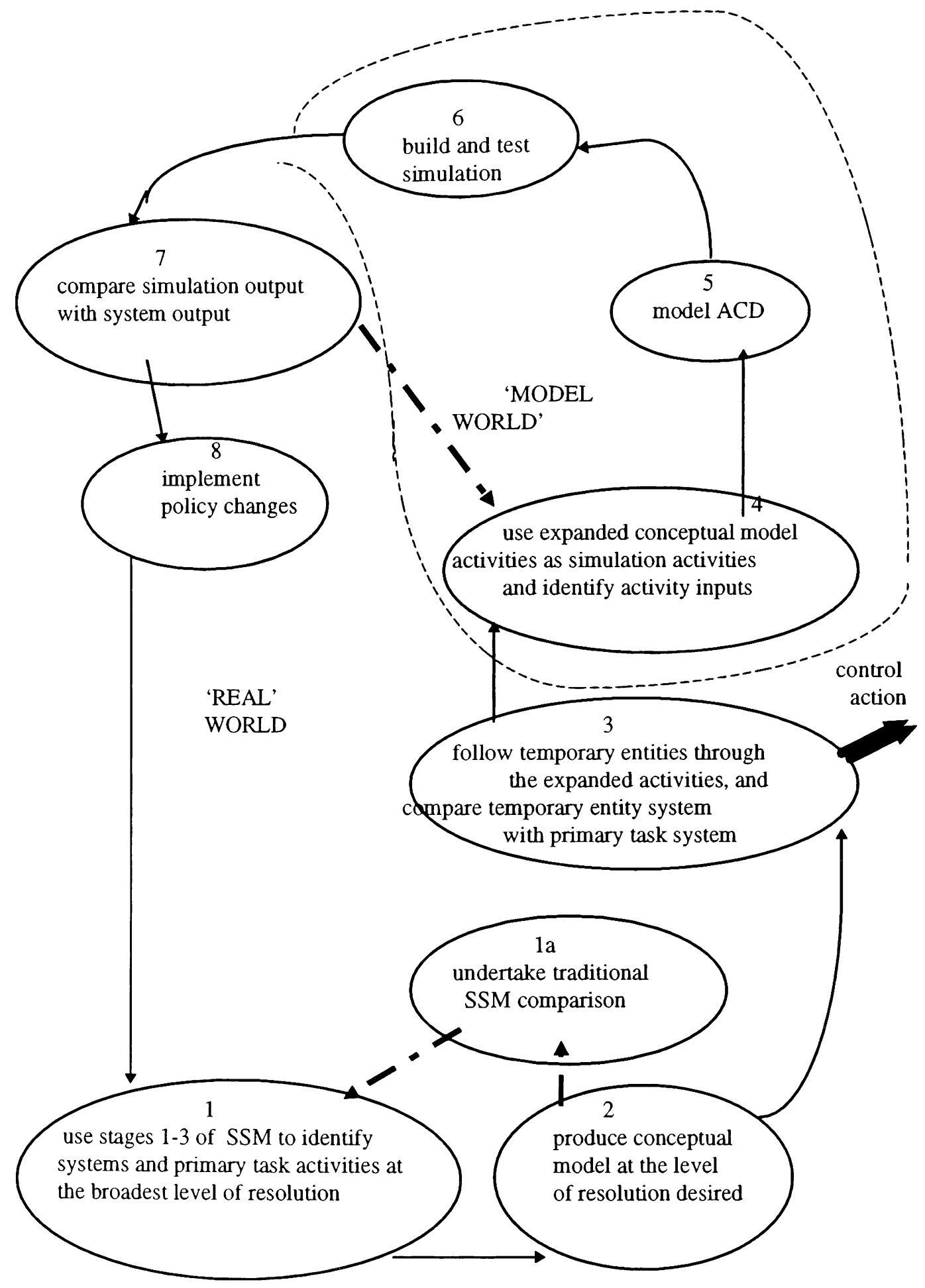

Figure 2: A Conceptual Combination of Simulation with SSM 
Table 1: Simulation Modelling Process

The detailed methodology outlined below should be accompanied by critical reviews of the overall project, its process, its timing, and its outcomes to-date, by means of scheduled meetings of analysts and stakeholders, and by any other means considered to be useful by the analysts and stakeholders. The frequency and duration of meetings may be adjusted as the project develops and in order to ensure that analysts and stakeholders have sufficient opportunity to cover any major issues relating to the project which they wish to raise. Some stages may be undertaken in a different order to that shown below, and some stages may be undertaken in parallel. The terms 'model' and 'system model' refer to diagrams, numbers, and words that describe the analysts' and stakeholders' views of the operations of the system (e.g., flowcharts). This is distinct from a 'computer simulation', although the latter will be based on a 'system model'.

A analysts FC first contact KS key stakeholders ORS other relevant stakeholders The First Contact below has been reassigned as a key stakeholder after the first two stages.

\section{$\underline{\text { Actors }} \quad \underline{\text { Actions }}$}

A, FC agree the broad nature and scope of the project

A, FC establish the initial key stakeholders

A, KS establish the broad area of investigation

A, KS re-establish the key stakeholders

A, KS establish the nature and scope of the project

A, KS agree working objectives

A, KS agree an initial project process and timetable

A, KS establish other relevant stakeholders

A, KS agree initial model of system boundaries and system activities

A build initial computer simulations

A run initial computer simulations using 'rough' data estimates

A, KS evaluate initial computer simulations

A, KS establish other relevant stakeholders

A, KS decide roughly how the system could be best examined 'on the ground'

A, KS, ORS inform ORS of the nature and scope of the project

$\mathrm{A}, \mathrm{KS}, \mathrm{ORS}$ refine the process of 'on the ground' system examination

$\mathrm{A}, \mathrm{KS}$,

A, KS, ORS

A, KS, ORS

A, KS

A, KS

A, KS

A

A

agree a timetable to examine the system 'on the ground'

examine the system 'on the ground'

collate information

refine the system model

refine the nature and scope of the project

refine the working objectives

refine the computer models

A, KS

run the refined computer models using 'rough' data estimates

$\mathrm{A}, \mathrm{KS}$

establish confidence in results

A, KS, ORS

A

refine data requirements

A, KS

collect and collate additional data

A, KS

run refined computer simulation using refined data

A, KS experiment with scenarios and analyse results

$\mathrm{KS}$

$\mathrm{KS}$

A, KS

A, KS determine the external and internal criteria which policy should address assess which policy options are both feasible and desirable establish how policy is to be implemented (practical, technical) implement policy assess the success of policy in meeting internal and external criteria reassess the nature and scope of the project 


\section{A QUICK AND DIRTY CASE}

A hospital outpatients department was the subject of a recent investigation. Table 1 shows the process. The discussions were essentially focus groups geared to patient throughflow. The key stakeholders were identified easily and very quickly. From this identification the rest follows.

Table 1 may appear to propose a sequential approach to a complex problem. In fact many of the activities in Table 1 may be undertaken in parallel, and the process is iterative. These activities can be grouped as a conceptual model, the root definition for which is as follows:

A system owned by a group of key stakeholders, run by analysts, key stakeholders, and other stakeholders, who use simulation modelling as an aid to develop and implement operational policy which meets internal and external criteria.

Using the CATWOE mnemonic:

Customer: unspecified

Actors: analysts, key and other

stakeholders

Transformation: to develop and

implement operational

policy

Weltanschaung: by using simulation

modelling as an aid,

operational policy may

be developed and

implemented

Owners: key stakeholders

Environment: internal and external criteria

The system which is being simulated is the outpatients department. The root definition and conceptual model here relate to a system of resolution which utilises that simulation model, but is not itself being simulated. If it were to be simulated (not impossible) then another overarching root definition and conceptual model for a system of resolution would be needed. The important point is that instead of the simulation being used simply to help solve a single specific problem at an operational level, it is now part of a system to resolve on-going complex situations at a strategic level.

The above process has been used successfully to develop a simulation of an outpatients department. The term 'successfully' is used here to denote that the end- users have welcomed the recommendations resulting from the investigation, and are taking control actions (patient rescheduling) accordingly. The above process is now seen as an important part of their monitoring and control procedures.

\section{CONCLUSIONS}

Simulation literature is sparse in the extreme with regard to process, and the principles of soft methods are useful for anyone planning or examining an investigation involving simulation.

SSM is conceptually the most appropriate soft approach to combine with simulation, but it is time consuming and unwieldy.

A quick and dirty approach to developing simulation models which utiilises the principles of soft methods may encourages end-user confidence in the early stages, and if an over-arching 'soft' framework is utilised, this confidence may be further fostered and maintained.

\section{REFERENCES}

Checkland, P.B. 1981. Systems Thinking, Systems Practice. Chichester: Wiley.

Mingers, J., and Taylor, S. 1992. The use of soft systems methodology in practice. Journal of the Operational Research Society. 43(4): 321-332.

Paul R.J., Balmer D. 1993. Simulation Modelling. Bromley: Chartwell Bratt.

Wilson B. 1984. Systems: Concepts, Methodologies, and Applications. Cichester: Wiley.

BRIAN LEHANEY is Principal Research Fellow in the Department of Business Systems at the University of Luton. His research focuses on simulation model development and building end-user confidence.

RAY J. PAUL is the first UK Professor of Simulation, and is also Head of the Department of Computing and Information Systems at Brunel University. Amongst other activities, his research centre (CASM) explores numerous aspects of discrete event simulation. 